\title{
DIE METAMORPHOSE IN DER URANINITFAMILIE
}

\author{
PAUL RAMDOHR
}

RAMDOHR, PAUL, 1986: Die Metamorphose in der Uraninitfamilie. Bull. Geol. Soc. Finland 58, Part 1, 263-269.

Die Uraninitfamilie wird im wesentlichen in zwei Untergruppen (Ausbildungsformen) mit gleichen Pulverdiagrammen gegliedert: der deutlich kubisch kristallisierte Uraninit und die traubig-nierige Pechblende, auch Uranpecherz oder Nasturan genannt.

Beispiele der Metamorphose in der Uraninitfamilie werden aus Europa (Norditalien, Schweiz und Bayern), Canada und Südafrika beschrieben und mittels Mikrophotographien illustriert. Aus allen Metamorphosebeobachtungen ist zu sehen, dass von der »Pronto-Reaktion» (Uraninit + Rutil = Brannerit) abgesehen, das Resultat der Veränderungen von Pechblenden immer ein fein- bis grobkörniger Uraninit ist - wie es ja eigentlich auch bei der Stabilität des Fluoritgitters von Uraninit zu erwarten ist.

\begin{abstract}
The uraninite family is subdivided into two subforms with identical powder patterns: a clearly cubic uraninite and a botryoidal-reniform »pitchblende» (nasturan).

Examples of metamorphism of uraninite from various occurrences in Europe (northern Italy, Switzerland and Bavaria), Canada and South-Africa are described and illustrated by photomicrographs. All observations on metamorphism show that, with the exception of the "pronto-reaction" (uraninite + rutile = brannerite), the endproduct of the transformations of the pitchblende always is a fine to coarse grained uraninite. This is, as a matter of fact, to be expected in view of the stability of the fluorite lattice of uraninite.
\end{abstract}

Key words: uraninite, pitchblende, metamorphism.

Paul Ramdohr, *1. 1. 1890 - †8. 3. 1985. The University of Heidelberg.

\section{Einleitung}

Die Uraninitfamilie wird mannigfach und zum Teil kompliziert untergegliedert. Im wesentlichen werden unterschieden: der deutlich kubisch kristallisierte Uraninit (z.B. der Pegmatite) und die typisch traubig-nierige Form der Pechblende, auch Uranpecherz oder Pecherz genannt, die von F. v. Kobell den Namen Nasturan (nach $\nu \alpha s \tau o ́ \zeta=$ dicht, derb) erhielt. Der lange vergessene Name ist neuerdings wieder belebt worden, vorwiegend im russischen Schrifttum. Das äussere Aussehen beider Hauptformen ist in der Tat so verschieden, dass zwei Benennungen durchaus berechtigt erscheinen. Im Pulverdiagramm sind beide aber völlig gleich, auch wenn die 


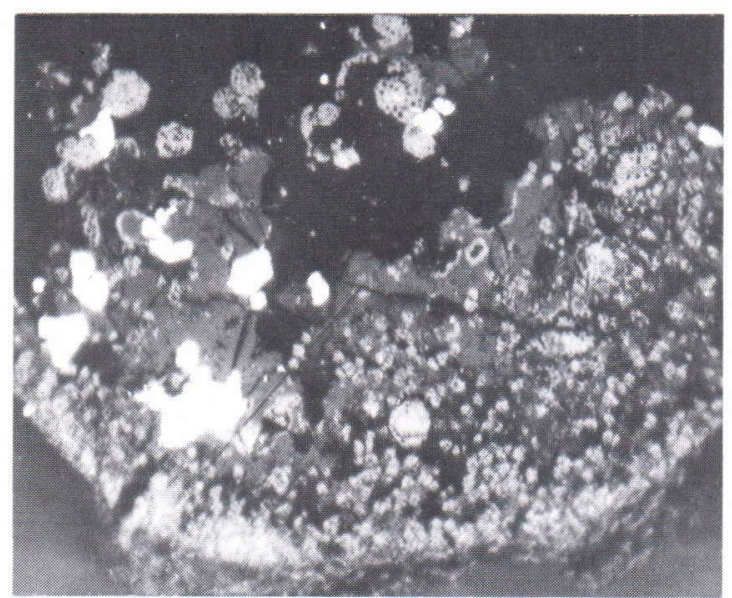

Fig. 1. Kolloidale Fällung von Pechblende in noch teilweise feinsttraubigem Zustand, durchwachsen mit Pyrit und vielleicht (gleichaussehend) Linnéit, Bocenago, Val Rendena, Norditalien $600 \mathrm{x}$.

chemische Zusammensetzung zwischen $\mathrm{UO}_{2}$ und $\mathrm{UO}_{2.7}$ etwa schwanken kann. Von kleinen Beimengungen, wie $\mathrm{ThO}_{2}$, mit dem bei hohen Temperaturen völlige Mischbarkeit besteht, $\mathrm{CeO}_{2}$ und einigen seltenen Erdoxyden sei hier abgesehen. Der Versuch, die lange Kette $\mathrm{UO}_{2}$ $\mathrm{UO}_{2.7} \mathrm{zu}$ unterteilen, z.B. "Nasturan I» bis »Nasturan IV», ist von Russen gemacht worden, wie es Verf. in seinem Buch (Ramdohr 1980, Abb. 601) ganz gut zeigt. In der Tat bestehen im Reflexionsvermögen recht deutliche Unterschiede, die aber im einzelnen nach ihren Ursachen nicht geklärt sind. Bisher sind diese Dinge besonders am »Nasturan» untersucht worden, obwohl auch bei wohlkristallinem »Uraninit» sehr starke Unterschiede im Verhältnis U : O bestehen, wie schon starker - nicht durch Th oder Ce-Gehalte bedingter Zonenbau bei beiden Varietäten zeigt.

Früher hat man der deutlich kristallinen Form meist hohe, der traubigen niedrige Bildungstemperatur zugeschrieben, was sicher roh stimmt, aber viele Ausnahmen hat. So sind z.B. in sonst völlig gleichartigen Paragenesen »Pechblenden» neben wohlkristallinem »Uraninit» oft beobachtet und es sind neuerdings in kanadischen Vorkommen wohlkristalline Würfelchen in grosser

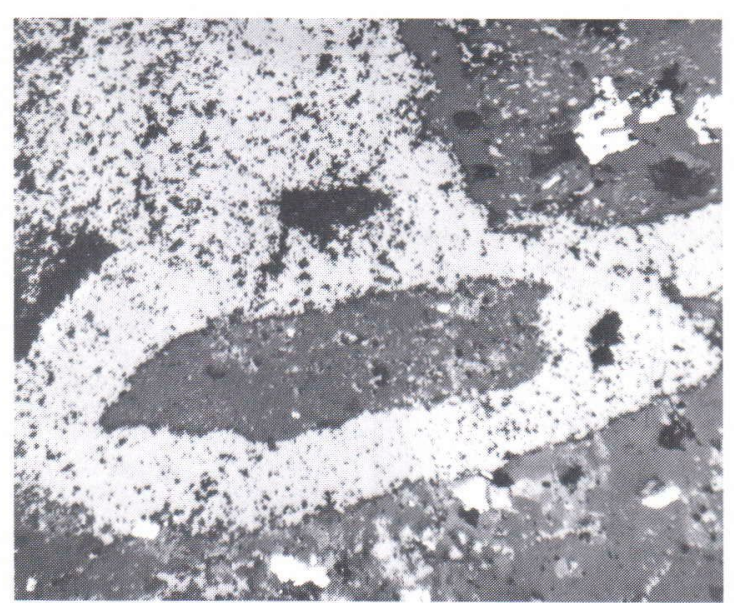

Fig. 2. In Uraninit vererzter Stengel einer Binse (Schrägschnitt). - Der eigentliche Kontakt zum Adamello-Granit ist hier noch erheblich weiter entfernt als in Alm Bos. Val Daone, Norditalien $70 \mathrm{x}$.

Menge gleichalterig neben sicher niedrig temperiertem Millerit $\left(<150^{\circ} \mathrm{C}\right)$ beschrieben worden. Dieser Uraninit hat in völlig frischem Zustand fast $\mathrm{U}_{3} \mathrm{O}_{7}$ (oder $\mathrm{UO}_{2.33}$ ). Dass gerade er meist deutlich aufgespaltene Pulverdiagrammlinien zeigt, die bestenfalls tetragonal, vielleicht nur noch niedriger symmetrisch deutbar sind, ist noch nicht recht verständlich. Dieser Uraninit ist meist deutlich anisotrop.

\section{Beispiele von verschiedenen Arten der Metamorphose}

Wie sich diese »Varietäten» verhalten, wenn sie den verschiedenen Arten der Metamorphose unterworfen wurden, ist recht wenig systematisch untersucht. Tatsächlich ist da ja eine grosse Gruppe von Uranlagerstätten, z.B. die weltgrösste vom »Colorado-Plateau-Typ» - im Colorado-Plateau selbst, Argentinien, mehrorts in der Sowjetunion, u.s.w. - auch die sehr alten des Gabuntyps und ebenso alle die berühmten Gangvorkommen wenig oder garnicht metamorphosiert. Über ein lokales Vorkommen allein, die alpinen Vorkommen, die letzten Endes dem permischen Vulkanismus, wie z.B. dem Bozener Quarzporphyr und seinen Tuffen entstam- 


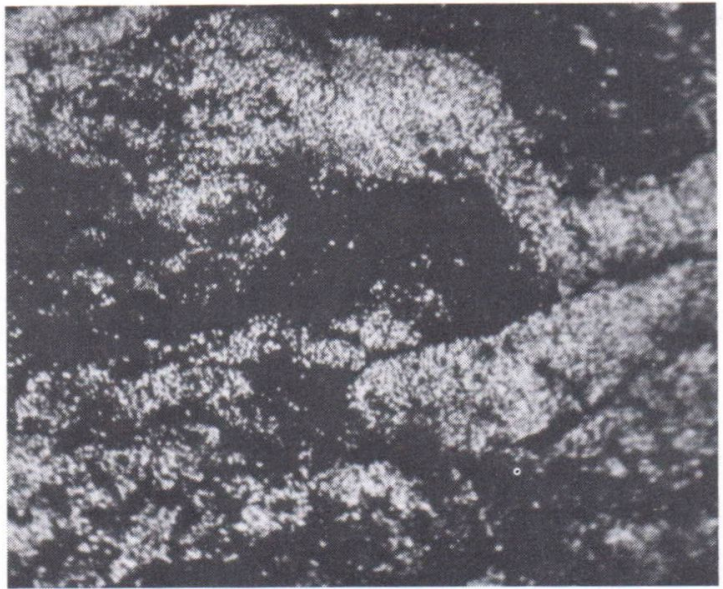

Fig. 3. Dicke Wolken fein-kristalliner Uraninit-Kristalle entstanden aus Uranpecherzschlamm. Pamparato-Grube, nö. Genua $250 \mathrm{x}$, Imm.

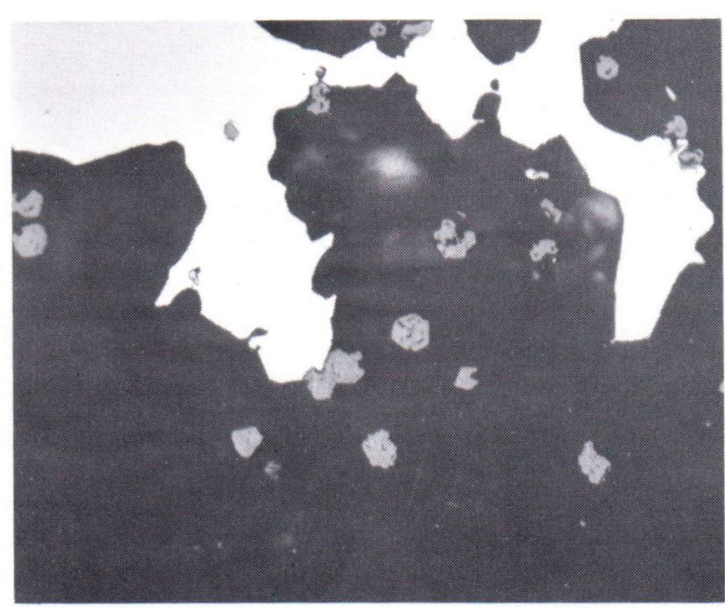

Fig. 5. Uraninit, deutliche Kristalle, die erheblich grösser als in Abb. 4 geworden sind. Sonst Gangart (schwarz) und Linnéit (weiss). Isérables, Wallis, Schweiz 250 x, Imm.

men und die verschiedenstufiger alpiner Metamorphose und der Kontaktmetamorphose des Adamello-Granits unterlegen sind, ist einiges gearbeitet und soll den Anfang meiner Darlegungen bilden. Sie haben z.B. aus Imprägnationen von erdigen Pechblenden im Gebiet von Preit und Pamparato (nördlich Genua) aus Quarzporphyrtuffen Quarzschiefer mit feinen Imprägnationen sehr feinkörnigen, aber stets sehr deut-

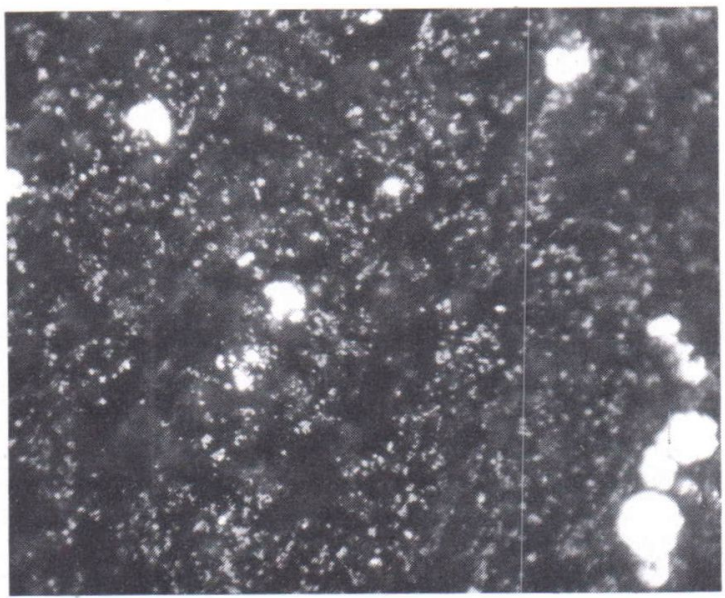

Fig. 4. Staub von feinen Uraninit-Kristallen (Würfelchen), die im Quarzphyllit aus feinstem Pechblendestaub entstanden sind. Preit, nö. Genua 600x.

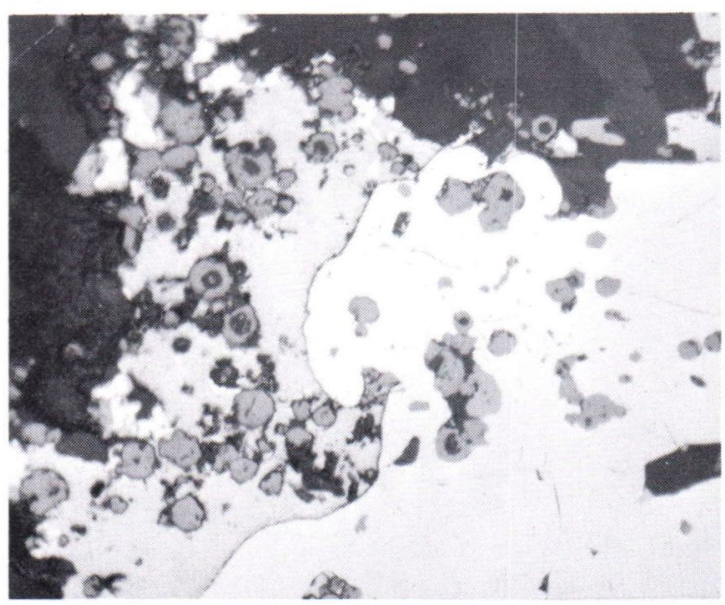

Fig. 6. Uraninit, z.T. deutliche Kristalle, teilweise rundlich mit zentralen Löchern. Im Kupferkies (grauweiss), neben Linnéit (reinweiss) und Gangarten (sehr dunkelgrau). Isérables, Wallis, Schweiz $300 \mathrm{x}$.

lich kristallinen Uraninit entstehen lassen (Abb. 3, 4). In der Epi- bis Mesozonalen Metamorphose der Penninischen Decken im schweizerischen Wallis (z.B. bei Isérables), sind bei sonst ähnlichen Verhältnissen die Uraninite schon deutlich gröber, aber immerhin noch im wesentlichen mikroskopische Objekte (Abb. 5, 6). Ähnliches gilt für Gesteine der nordwestlichen Umgebung von Chur. 


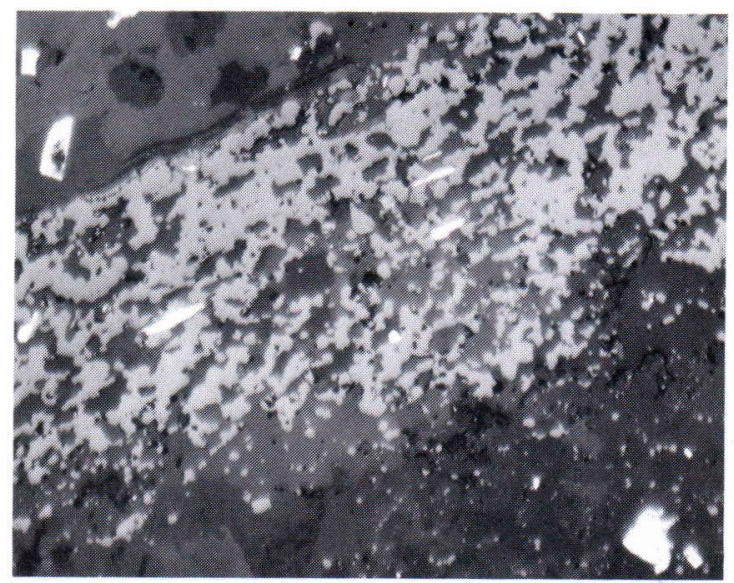

Fig. 7. Aggregat von vererztem Holz. Der Uraninit sitzt wie schon am ganz unveränderten Erz - bevorzugt auf der Oberfläche des Holzes, dessen Grenze noch deutlich erkennbar ist. - Die Si-Gangartmineralien sind z.T. noch unbestimmte V-haltige Silikate (rechts oder oben). Alm Bos, $150 \mathrm{x}$.

Wie sich Gesteine, die noch in dem Bozener Quarzporphyr im nahe gelegenen Val Judicaria bei Bocenago praktisch unverändert den Coloradoplateautyp zeigen (Abb. 1, 2) mit Reduktion und Fällung des Urangehaltes auf Pflanzenhäcksel, Binsenresten und Holzstückchen verhalten, wenn sie in den Kontakthof des ganz jungen Tonalegranits bei der Alm Bos gelangen (Ramdohr op. cit. Lit), (Abb. 7, 8, 9) konnte Verf. mit G. Cevales vor kurzem zeigen. Die kompakten Pechblenden sind wieder in hier recht grobkörnigen Uraninit verwandelt, wobei aber z.B. Pflanzenreste, z.B. Binsen noch gut erkennbar sind. Interessant sind gerade hier die Reaktionen einiger Begleitelemente, wie Vanadin, Kobalt und Nickel. Dass auch bei der höchsten Metamorphose die »Stöchiometrie» $\mathrm{UO}_{2}$ noch nicht erreicht ist, scheint die auch hier noch bestehende Anisotropie zu zeigen.

Eine ganz überraschende Metamorphosereaktion zeigt nun eine neuerdings erkannte Paragenese. In der Gegend von Poppenreuth (in der Bayer. Oberpfalz) kommen uralte, wohl assyntische Uranerze vor, die vielleicht einem »Colorado-Plateau-Typ» entsprechen. Das Material ist irgendwie hydrothermal umgelagert und

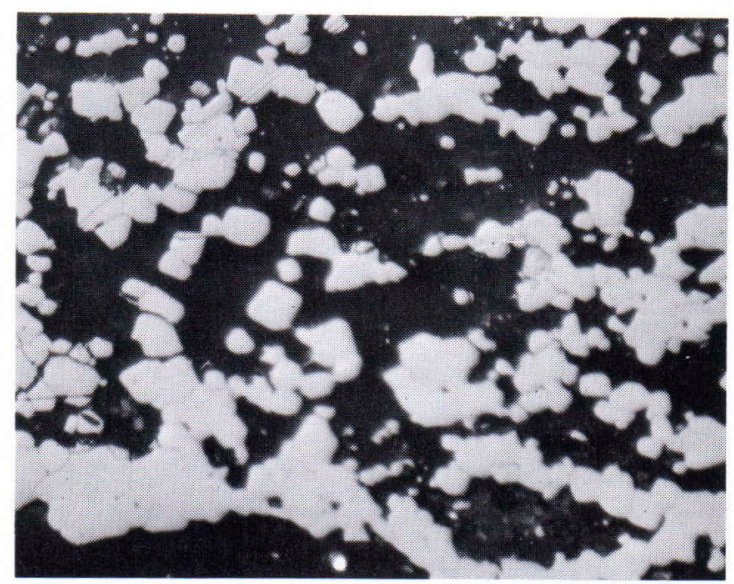

Fig. 8. Einzelheit aus dem Bild der vererzten Binse. Die Uraninit-Kristalle zeigen ausser dem Würfel z.T. auch das Oktaeder. Val Daone, Norditalien $250 \mathrm{x}$, Imm.

hat kleine Vorkommen von wohlentwickeltem traubigem Uranpecherz geliefert. Erneute Durchbewegung liess diese Bildungen zerbrechen in eine Erz-Nebengesteinsbreccie, in der mit freiem Auge (Abb. 10, nat. Grösse) die traubigen Pecherzmassen noch ohne weiteres erkennbar sind. Bei mikroskopischer Untersuchung ergeben sich eigentümliche Verhältnisse: Partien, die nicht stark gedrückt, sondern eher gedehnt waren, behalten einigermassen den alten Charakter. Sie können sogar (Abb. 11) aufgelockert erscheinen. Sind sie dagegen gepresst, so setzt eine feinkörnige Rekristallisation ein, die ein ganz gleichmässiges Aggregat rundlich polygonaler Uraninitkörnchen liefert (Abb. 12). Wenn die Aggregate ganz leichter beginnender Verwitterung ausgesetz waren, so sind, ohne jede Rücksicht auf die alten, wie gesagt, makroskopisch noch gut erkennbaren Strukturen, die Einzelkörnchen zonar etwas angewittert mit leicht wechselndem Reflexionsvermögen. Jedenfalls ist die feinkörnige Rekristallisation auch so ganz augenfällig gemacht (Abb. 12). Noch spätere Beanspruchung kann die Aggregate der feinen Körnchen etwas zerreiben oder verteilen, wobei Silikatmineralien und Sulfide einwandern (Abb. 13). 


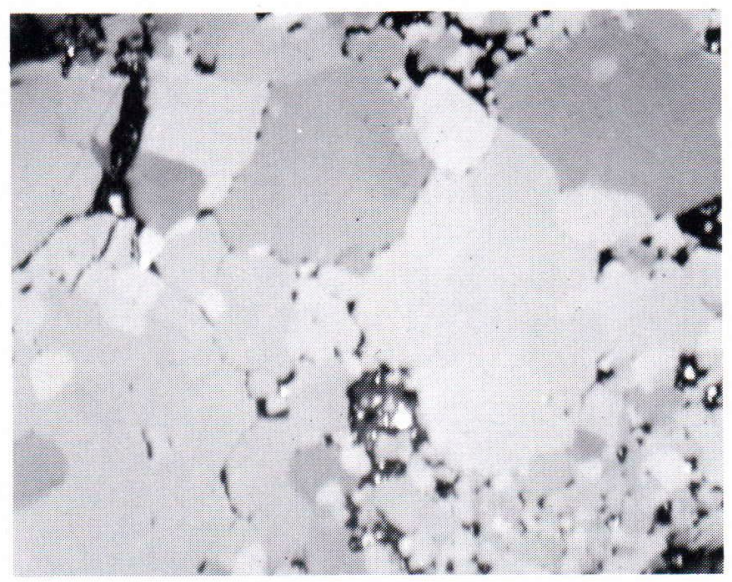

Fig. 9. Der durch Kontakt metamorphosierte Uraninit zeigt neben gelegentlich guten Kristallen auch sehr deutliche Anisotropie. Alm Bos, Val Camonica, 325 x, Imm., Nic. fast gekreuzt.

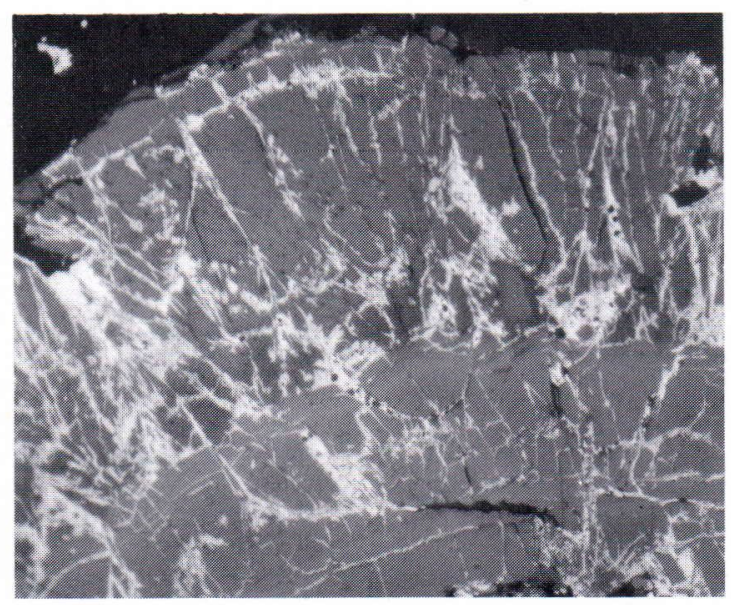

Fig. 11. Traubig-schalige Pechblende in der Längsrichtung etwas gezerrt. Hier nichts von Rekristallisation erkennbar, feine Durchäderung mit Kupferkies. Höhenstein b. Poppenreuth, Bayern $180 \mathrm{x}$.

Da die komplizierten Voraussetzungen für diese Bildungen recht selten gegeben sind, ist dieses Rekristallisat wohl als recht ungewöhnlich anzusehen und wird zudem leicht übersehen.

\section{Die Reaktion Uraninit + Rutil $=$ Brannerit}

Nicht erwähnt war bisher die »ProntoReaktion», die durch anscheinend einfache Über-

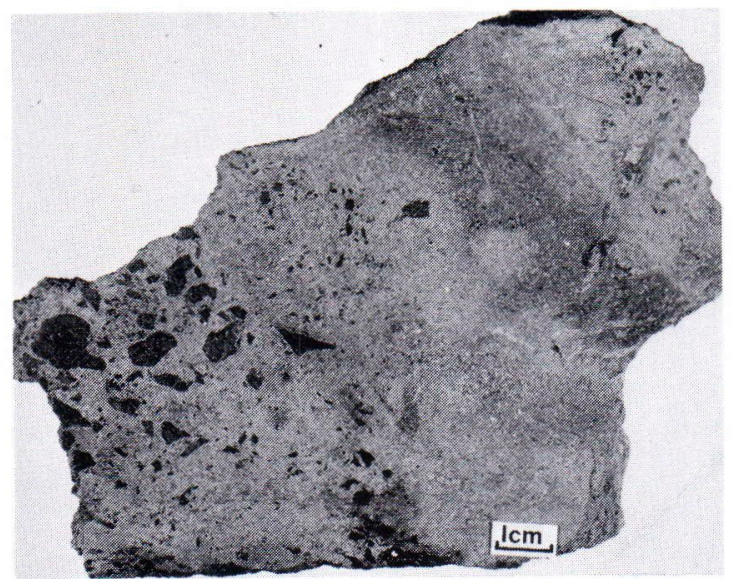

Fig. 10. Umgelagerte, stark zerbrochene, traubige Pechblende, schwarz, in ebenfalls zerriebenem Nebengestein. Natürliche Grösse. Höhenstein-Grube b. Poppenreuth, Bayern.

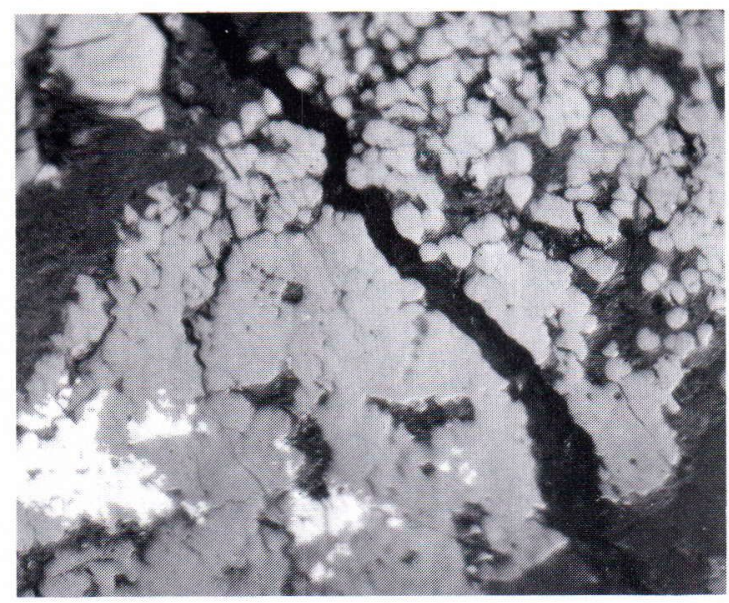

Fig. 12. Pechblende, feinkörnig, zu Uraninit rekristallisiert. Hier nicht verwittert, so dass Korngrenzen in kompakten Partien unsichtbar sind. Höhenstein-Grube b. Poppenreuth, Bayern $180 \mathrm{x}$.

lagerungsmetamorphose bedingte Reaktion Uraninit + Rutil zu Brannerit $\left(\mathrm{UO}_{2}+2 \mathrm{TiO}_{2}=\right.$ $\mathrm{UTi}_{2} \mathrm{O}_{6}$ ), einen Vorgang, den man in geschmolzenem $\mathrm{NaCl}$ bei rund $800^{\circ} \mathrm{C}$ ausgezeichnet in 24 Stunden zu einem wohlkristallinen Produkt nachahmen kann, der aber in polynären Chloridschmelzen schon bei viel niedriger Temperatur (vielleicht $300^{\circ} \mathrm{C}$ ), allerdings erheblich lang- 


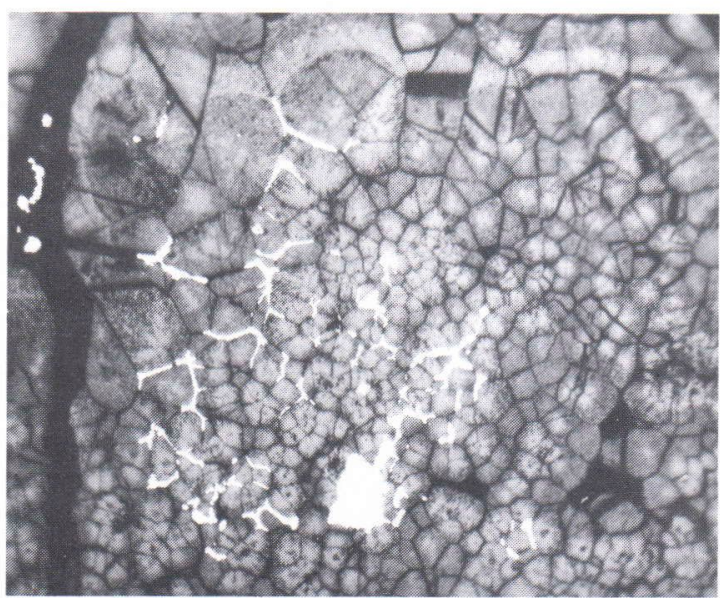

Fig. 13. Pechblende, durch Druck feinkörning rekristallisiert, randlich noch Andeutung der traubigen Struktur erkennbar. Dieses Rekristallisat ist leicht durch Anwitterung erkennbar. - Kontraste photographisch betont. Stellenweise Durchäderung mit Kupferkies. Höhenstein-Grube b. Poppenreuth, Bayern $300 \mathrm{x}$.

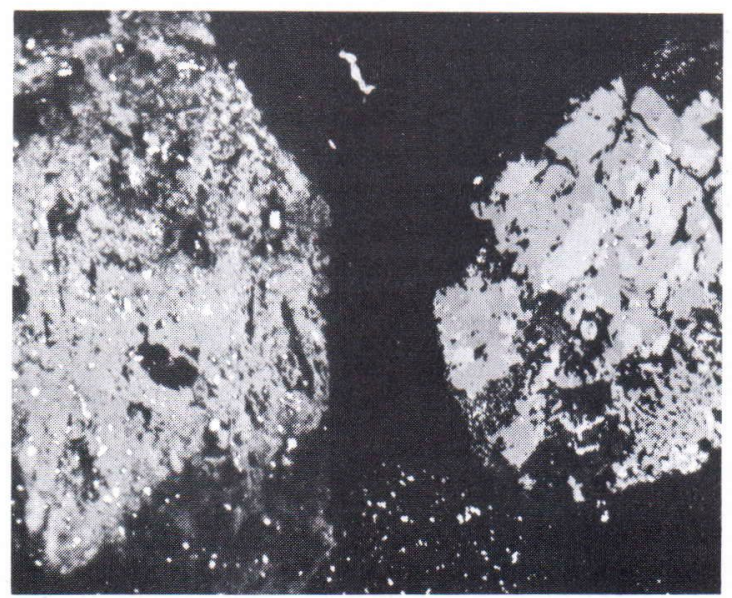

Fig. 15. Pseudomorphosen nach Ilmenit, rechts im wesentlichen Aggregat von Rutil, links Aggregat von Brannerit. Afrikander-Mine, Dominion Reef, Südafrika 200 x, Imm.

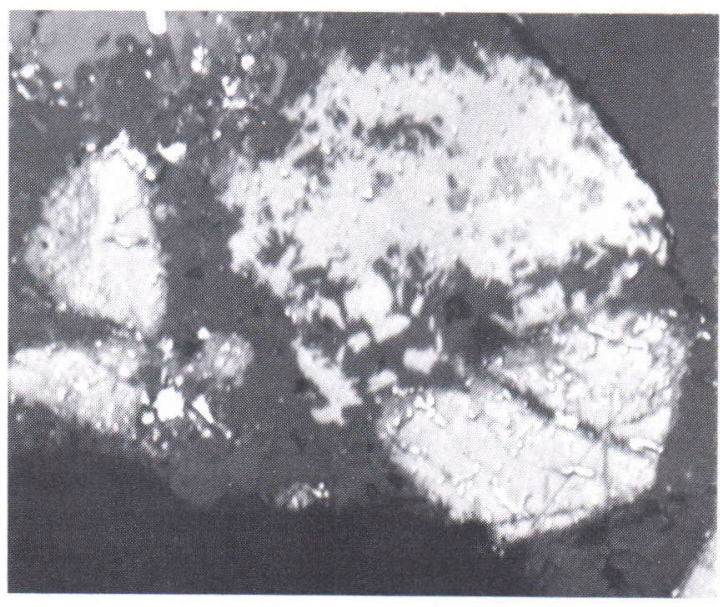

Fig. 14. Uraninitkorn, das zur Hälfte (oben) in Brannerit verwandelt ist, zur Hälfte (unten links) noch intakt ist mit guter Spaltbarkeit //(111). Schwarz ist Quarz, dunkelgrau andere Gangarten. Pronto Mine, nö. Huron Lake, Canada $450 \mathrm{x}, \mathrm{Imm}$.

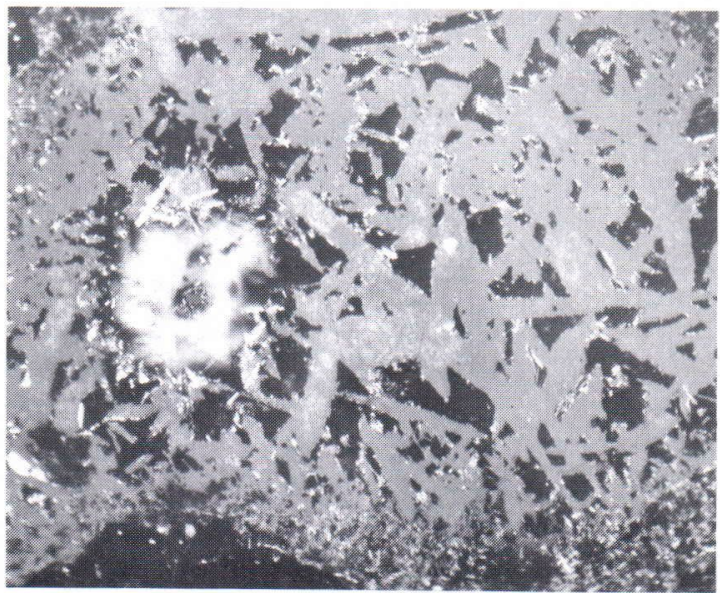

Fig. 16. Brannerit-Kristalle, entstanden durch Wechselreaktion des ehemaligen Ilmenitskeletts (davon Struktur) mit Uraninit. Kleinkörniger Staub von Anatas, vereinzelte Lamellen von dünntafeligem Pyrrhotin. Pronto-mine, Canada $300 \mathrm{x}$, Imm.

Wenn die Reaktion sich vollzogen hat, setzt mit zunehmendem Uranzerfall eine Ausscheidung von feinkörnigem Anatas ein, da ja dann in der Branneritform nicht alles Ti mehr seinen Uranpartner besitzt. Mann könnte - es ist bisher noch nie versucht worden - aus dem Anatasgehalt des Brannerits den Zeitpunkt der »Pronto-Reaktion» Blind River - Elliot-Lake u.ä. beschränkt (Abb. 14, 15, 16, 17, 18). 


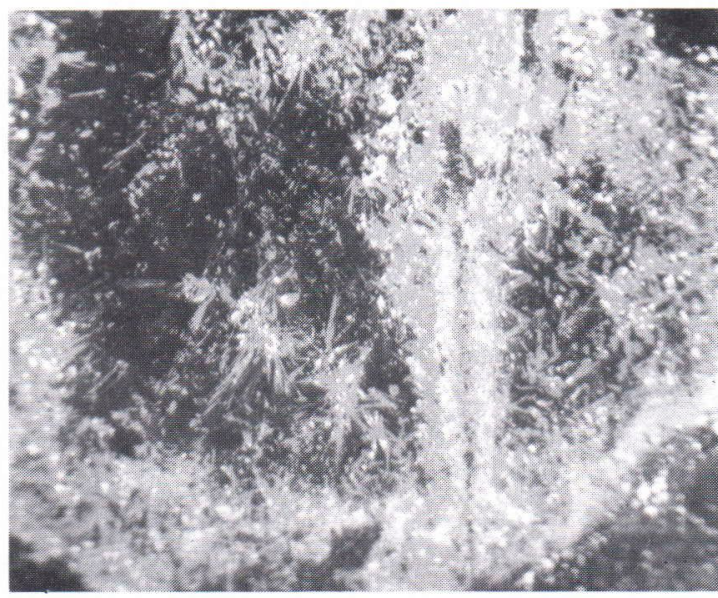

Fig. 17. Ein ehemaliger Titanomagnetit ist über Rutil in Nadeln von Brannerit verwandelt. Bramley Mine, Dominion Reef, Südafrika 250 x, Imm.

feststellen. Zurückbildung von Rutil statt Anatas ist nie beobachtet worden.

\section{Schlusswort}

Insgesamt ist aus allen Metamorphosebeobachtungen zu sehen, dass von der weltweiten, besondere Voraussetzungen verlangenden »ProntoReaktion» abgesehen, das Resultat der Verände-

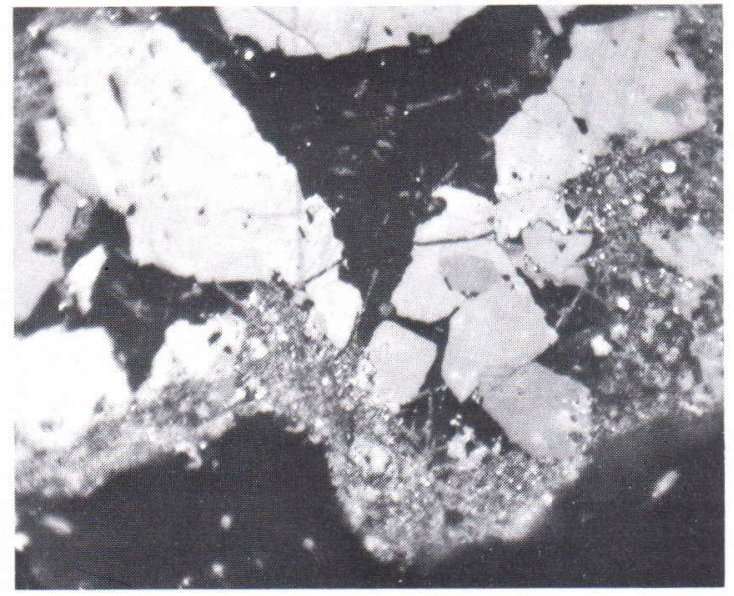

Fig. 18. Feine Nädelchen von Brannerit verdrängen recht grobkörnigen Rutil. Afrikander Mine, Dominion Reef, Südafrika. $600 \mathrm{x}$, Imm.

rung von »Pechblenden» immer ein fein- bis grobkörniger Uraninit ist - wie es ja eigentlich auch bei der Stabilität des Fluoritgitters von Uraninit zu erwarten ist.

\section{Literatur}

Ramdohr, P., 1980. The Ore Minerals and Their Intergrowths. Pergamon Press Oxford und Akademie-Verlag, Berlin. 1207 p. 